\title{
Robust transmission stabilization and dynamic switching in broadband hybrid waveguide systems with nonlinear gain and loss
}

\author{
Quan M. Nguyen ${ }^{1}$, Avner Peleg ${ }^{2}$, and Thinh P. Tran ${ }^{3}$ \\ ${ }^{1}$ Department of Mathematics, International University, \\ Vietnam National University-HCMC, Ho Chi Minh City, Vietnam \\ ${ }^{2}$ Department of Mathematics, State University of New York at Buffalo, \\ Buffalo, New York 14260, USA and \\ ${ }^{3}$ Department of Theoretical Physics, University of Science, \\ Vietnam National University-HCMC, Ho Chi Minh City, Vietnam
}

(Dated: August 11, 2018)

\begin{abstract}
We develop a method for transmission stabilization and robust dynamic switching for colliding optical soliton sequences in broadband waveguide systems with nonlinear gain and loss. The method is based on employing hybrid waveguides, consisting of spans with linear gain and cubic loss, and spans with linear loss, cubic gain, and quintic loss. We show that amplitude dynamics is described by a hybrid Lotka-Volterra (LV) model, and use the model to determine the physical parameter values required for enhanced transmission stabilization and switching. Numerical simulations with the coupled nonlinear Schrödinger equations confirm the predictions of the LV model, and show complete suppression of radiative instability, which enables stable transmission over distances larger by an order of magnitude compared with uniform waveguides with linear gain and cubic loss. Moreover, multiple on-off and off-on dynamic switching events are demonstrated over a wide range of soliton amplitudes, showing the superiority of hybrid waveguides compared with static switching in uniform waveguides.
\end{abstract}

PACS numbers: 42.65.Tg, 42.81.Dp, 42.65.Sf 


\section{INTRODUCTION}

Recent years have seen a dramatic increase in research on broadband optical waveguide systems [1 4]. This increase in research efforts is driven by a wide range of applications, which include increasing transmission rates in fiber optics communication systems [2 4] , enhancing data processing and transfer on computer chips [5-8], and enabling multiwavelength optical waveguide lasers [9 14]. Transmission in broadband systems is often based on wavelength-division-multiplexing (WDM), where many pulse sequences propagate through the same waveguide. The pulses in each sequence (each "frequency channel") propagate with the same group velocity, but the group velocity differs for pulses from different sequences. As a result, intersequence pulse collisions are very frequent, and can lead to severe transmission degradation [1, 2, 4, 15, 16]. On the other hand, the significant collision-induced effects can be used for controlling the propagation, for tuning of optical pulse parameters, such as energy, frequency, and phase, and for transmission switching, i.e., the turning on or off of transmission of one or more of the pulse sequences [17-19].

One of the most important processes affecting pulse propagation in nonlinear waveguide systems is due to nonlinear loss or gain. Nonlinear loss (gain) can arise in optical waveguides due to multiphoton absorption (emission) or due to gain (loss) saturation [20, 21]. For example, cubic loss due to two-photon absorption (TPA) plays a key role in pulse dynamics in a variety of waveguides, including silicon waveguides [5 8, 22 32]. Furthermore, cubic gain and quintic loss are essential parts of the widely used Ginzburg-Landau (GL) model for pulse dynamics in mode-locked lasers [33-38]. The main effect of nonlinear loss (gain) on single pulse propagation is a continuous decrease (increase) of the pulse amplitude, which is qualitatively similar to the one due to linear loss (gain) [22]. Nonlinear loss (gain) also strongly affects optical pulse collisions, by causing an additional decrease (increase) of pulse

amplitudes [17-19, 39]. This collision-induced amplitude shift, which is commonly known as interchannel crosstalk, can be a major impairment in broadband nonlinear waveguide systems. For example, recent experiments have shown that crosstalk induced by cubic loss (due to TPA) plays a key role in silicon nanowaveguide WDM systems [32]. More specifically, the experiments demonstrated that TPA-induced crosstalk can lead to relatively high values of the bit-error-rate even in a WDM system with two channels [32]. Thus, it is important to find ways to suppress the detrimental effects of nonlinear gain-loss crosstalk. 
In several recent studies [17 19] we provided a partial solution to this key problem and to an equally important challenge concerning the possibility to use the nonlinear crosstalk for broadband transmission switching. Our approach was based on showing that amplitude dynamics of $\mathrm{N}$ sequences of colliding optical solitons can be described by Lotka-Volterra (LV) models for $\mathrm{N}$ species, where the exact form of the LV model depends on the nature of the waveguide's gain-loss profile [17, 18]. Stability analysis of the steady states of the LV models was used to guide a clever choice of linear amplifier gain, which in turn leads to transmission stabilization, i.e., the amplitudes of the propagating pulses approach desired predetermined values [17-19]. Furthermore, in Ref. [19], we showed that static on-off and offon transmission switching can be realized by an abrupt change in the waveguide's nonlinear gain or loss coefficients. The design of the switching setups reported in Ref. [19] was also guided by linear stability analysis of the steady states of the LV model.

The results of Refs. [17-19] demonstrate the potential of employing crosstalk induced by nonlinear loss or gain for transmission control, stabilization, and switching. However, these results are still quite limited due to the following reasons. First, despite the progress made in Refs. [17-19], the problem of robust transmission stabilization is still unresolved. In particular, for uniform waveguides with linear gain and cubic loss, such as silicon waveguides, radiative instability due to the growth of small amplitude waves is observed already at a distance $z \simeq 200$ even for cubic loss coefficient values as small as 0.01 [17]. The radiative instability can be partially mitigated by employing uniform waveguides with linear loss, cubic gain, and quintic loss, i.e., waveguides with a GL gain-loss profile [18, 19]. However, this uniform GL gain-loss setup is also limited, since the initial soliton amplitudes need to be close to the steady state values for transmission stabilization to be achieved. Second, the switching setup studied in Ref. [19] is also quite limited, since it is based on a static change in the waveguide's nonlinear gain-loss coefficients. Moreover, only one switching event was demonstrated in this study, and off-on transmission was restricted to amplitude values larger than 0.65 . In view of the limitations of these uniform waveguide setups, it is important to look for more robust ways for realizing stable long-distance propagation and broadband transmission switching.

In the current paper we take this important task, by developing a method for transmission stabilization and switching in broadband waveguide systems, which is based on employing hybrid waveguides with a clever choice of the physical parameters. The hybrid waveguides 
consist of odd-numbered spans with linear gain and cubic loss, and even-numbered spans with a GL gain-loss profile. Transmission switching is dynamically realized by fast changes in linear amplifier gain. The robustness of the approach is demonstrated for two sequences of colliding optical solitons. We show that the dynamics of soliton amplitudes is described by a hybrid LV model. We then use stability analysis for the steady states of the LV model to determine the physical parameters that lead to suppression of radiative instability and as a result, to a drastic enhancement in transmission stability and switching robustness. The hybrid nature of the waveguides and the corresponding LV model plays a key role in the improvement. The predictions of the hybrid LV model are confirmed by numerical simulations with the full system of coupled nonlinear Schrödinger (NLS) equations. The results of the latter simulations show complete suppression of radiative instability, which enables stable propagation over distances larger by an order of magnitude compared with the results reported in Ref. [17] for uniform waveguides with linear gain and cubic loss. Moreover, multiple dynamic on-off and off-on switching events are demonstrated over a significantly wider range of soliton amplitudes compared with that reported in Ref. [19] for a single static switching event in uniform waveguides with a GL gain-loss profile. The increased robustness of off-on switching in hybrid waveguides can be used for transmission recovery, that is, for the stable amplification of optical pulse sequences that experienced significant energy decay.

We choose optical solitons as an example for the propagating pulses for the following reasons. First, in many broadband optical systems the waveguides are nonlinear and pulse propagation is accurately described by a perturbed NLS equation [6 8, 24, 25, 27]. Furthermore, optical soliton generation and propagation in the presence of two-photon and three-photon absorption was experimentally demonstrated in a variety of waveguide setups [29, 30, 40 43]. Second, since the unperturbed NLS equation is an integrable model [44], derivation of analytic results for the effects of nonlinear gain or loss on interpulse collisions can be done in a rigorous manner. Third, due to the soliton properties, soliton-based information transmission and processing in nonlinear broadband waveguide links is considered to be highly advantageous compared with other transmission methods [1, 2, 16].

The rest of the paper is organized as follows. In Sec. III, we present the coupled-NLS model for pulse propagation in hybrid waveguides, along with the corresponding hybrid LV model for amplitude dynamics. We then use stability analysis of the equilibrium states of 
the hybrid LV model to obtain the physical parameter values required for robust transmission stabilization and broadband switching. In Sec. III, we present the results of numerical simulations with the coupled-NLS model for stable long-distance propagation and multiple transmission switching events. We also analyze these results in comparison with the predictions of the LV model. Section [V] is reserved for conclusions.

\section{COUPLED-NLS AND LOTKA-VOLTERRA MODELS FOR PULSE PROPA- GATION}

We consider two sequences of optical solitons propagating with different group velocities in a hybrid waveguide system, in which the gain-loss profile is different for different waveguide spans. We take into account second-order dispersion, Kerr nonlinearity, as well as linear and nonlinear gain and loss. We denote by $z$ distance along the waveguide, and assume that the gain-loss profile consists of linear gain and cubic loss in odd-numbered spans $z_{2 m} \leq z<$ $z_{2 m+1}$, and of linear loss, cubic gain, and quintic loss in even-numbered spans $z_{2 m+1} \leq z<$ $z_{2 m+2}$, where $0 \leq m \leq M, M \geq 0$, and $z_{0}=0$. Thus, the propagation is described by the following system of coupled-NLS equations:

$$
i \partial_{z} \psi_{j}+\partial_{t}^{2} \psi_{j}+2\left|\psi_{j}\right|^{2} \psi_{j}+4\left|\psi_{k}\right|^{2} \psi_{j}=i g_{j}^{(l)} \psi_{j} / 2+L_{l}\left(\psi_{j}, \psi_{k}\right)
$$

where $t$ is time, $\psi_{j}$ is the electric field's envelope for the $j$ th sequence, $g_{j}^{(l)}$ is the linear gain-loss coefficient, and $L_{l}\left(\psi_{j}, \psi_{k}\right)$ describes nonlinear gain-loss effects. The indexes $j$ and $k$ run over pulse sequences, i.e., $j=1,2, k=1,2$, while $l$ runs over the two gain-loss profiles. The second term on the left hand side of Eq. (11) corresponds to second-order dispersion, while the third and fourth terms describe the effects of intrasequence and intersequence interaction due to Kerr nonlinearity.

The optical pulses in the $j$ th sequence are fundamental solitons of the unperturbed NLS equation $i \partial_{z} \psi_{j}+\partial_{t}^{2} \psi_{j}+2\left|\psi_{j}\right|^{2} \psi_{j}=0$. The envelopes of these solitons are given by $\psi_{s j}(t, z)=$ $\eta_{j} \exp \left(i \chi_{j}\right) \operatorname{sech}\left(x_{j}\right)$, where $x_{j}=\eta_{j}\left(t-y_{j}-2 \beta_{j} z\right), \chi_{j}=\alpha_{j}+\beta_{j}\left(t-y_{j}\right)+\left(\eta_{j}^{2}-\beta_{j}^{2}\right) z$, and $\eta_{j}, \beta_{j}, y_{j}$, and $\alpha_{j}$ are related to the soliton amplitude, group velocity (and frequency), position, and phase, respectively. We assume a large group velocity difference $\left|\beta_{1}-\beta_{2}\right| \gg$ 1, so that the solitons undergo a large number of fast intersequence collisions. Due to the presence of nonlinear gain or loss the solitons experience additional changes in their 
amplitudes during the collisions, and this can be used for achieving robust transmission stabilization and switching.

The nonlinear gain-loss term $L_{1}\left(\psi_{j}, \psi_{k}\right)$ in odd-numbered spans is

$$
L_{1}\left(\psi_{j}, \psi_{k}\right)=-i \epsilon_{3}^{(1)}\left|\psi_{j}\right|^{2} \psi_{j}-2 i \epsilon_{3}^{(1)}\left|\psi_{k}\right|^{2} \psi_{j}
$$

where $\epsilon_{3}^{(1)}$ is the cubic loss coefficient. The first and second terms on the right hand side of Eq. (2) describe intrasequence and intersequence interaction due to cubic loss. The nonlinear gain-loss term $L_{2}\left(\psi_{j}, \psi_{k}\right)$ in even-numbered spans is

$$
\begin{aligned}
& L_{2}\left(\psi_{j}, \psi_{k}\right)=i \epsilon_{3}^{(2)}\left|\psi_{j}\right|^{2} \psi_{j}+2 i \epsilon_{3}^{(2)}\left|\psi_{k}\right|^{2} \psi_{j} \\
& -i \epsilon_{5}\left|\psi_{j}\right|^{4} \psi_{j}-3 i \epsilon_{5}\left|\psi_{k}\right|^{4} \psi_{j}-6 i \epsilon_{5}\left|\psi_{k}\right|^{2}\left|\psi_{j}\right|^{2} \psi_{j},
\end{aligned}
$$

where $\epsilon_{3}^{(2)}$ and $\epsilon_{5}$ are the cubic gain and quintic loss coefficients, respectively. The first and second terms on the right hand side of Eq. (3) describe intrasequence and intersequence interaction due to cubic gain, while the the third, fourth, and fifth terms are due to quintic loss effects.

In several earlier works, we showed that amplitude dynamics of $N$ colliding sequences of optical solitons in the presence of linear and nonlinear gain or loss can be described by LV models for $N$ species, where the exact form of the model depends on the nature of the waveguide's gain-loss profile [17, 18, 45]. The derivation of the LV models was based on the following assumptions. (1) The temporal separation $T$ between adjacent solitons in each sequence is a constant satisfying: $T \gg 1$. In addition, the amplitudes are equal for all solitons from the same sequence, but are not necessarily equal for solitons from different sequences. This setup corresponds, for example, to return-to-zero phase-shift-keyed soliton transmission. (2) The pulses circulate in a closed optical waveguide loop. (3) As $T \gg 1$, the pulses in each sequence are temporally well-separated. As a result, intrasequence interaction is exponentially small and is neglected.

Under the above assumptions, the soliton sequences are periodic, and as a result, the amplitudes of all pulses in a given sequence undergo the same dynamics. Consider first oddnumbered waveguide spans, where the gain-loss profile consists of linear gain and cubic loss. Taking into account collision-induced amplitude shifts due to cubic loss and single-pulse amplitude changes due to linear gain and cubic loss, we obtain the following equation for 
amplitude dynamics of $j$ th sequence solitons [17]:

$$
\frac{d \eta_{j}}{d z}=\eta_{j}\left(g_{j}^{(1)}-4 \epsilon_{3}^{(1)} \eta_{j}^{2} / 3-8 \epsilon_{3}^{(1)} \eta_{k} / T\right)
$$

where $j=1,2$ and $k=1,2$. In WDM transmission systems, it is often required to achieve a transmission steady state, in which pulse amplitudes in all sequences are nonzero constants. We therefore look for a steady state of Eq. (44) in the form $\eta_{1}^{(e q)}=a>0, \eta_{2}^{(e q)}=b>0$, where $a$ and $b$ are the desired equilibrium amplitude values. This requirement yields: $g_{1}^{(1)}=$ $4 \epsilon_{3}^{(1)}\left(a^{2} / 3+2 b / T\right)$ and $g_{2}^{(1)}=4 \epsilon_{3}^{(1)}\left(b^{2} / 3+2 a / T\right)$. Note that in transmission stabilization and off-on switching we use $a=b=\eta$, corresponding to the desired situation of equal amplitudes in both sequences. In contrast, in on-off switching, we use $a \neq b$, since turning off of transmission of only one sequence is difficult to realize with $a=b$. Also note that switching is obtained by fast changes in the values of the $g_{j}^{(1)}$ coefficients, such that $(a, b)$ becomes asymptotically stable in off-on switching and unstable in on-off switching. The switching is realized dynamically, via appropriate fast changes in amplifier gain, and is thus very different from the static switching that was studied in Ref. [19].

The LV model for amplitude dynamics in even-numbered spans is obtained by taking into account collision-induced amplitude shifts due to cubic gain and quintic loss, as well as single-pulse amplitude changes due to linear loss, cubic gain, and quintic loss. The derivation yields the following equation for amplitude dynamics of the $j$ th sequence solitons [18]:

$$
\frac{d \eta_{j}}{d z}=\eta_{j}\left[g_{j}^{(2)}+4 \epsilon_{3}^{(2)} \eta_{j}^{2} / 3-16 \epsilon_{5} \eta_{j}^{4} / 15+8 \epsilon_{3}^{(2)} \eta_{k} / T-8 \epsilon_{5} \eta_{k}\left(2 \eta_{j}^{2}+\eta_{k}^{2}\right) / T\right]
$$

Requiring that $(\eta, \eta)$ is a steady state of Eq. (15), we obtain $g_{j}^{(2)}=4 \epsilon_{5} \eta\left(-\kappa \eta / 3+4 \eta^{3} / 15-\right.$ $\left.2 \kappa / T+6 \eta^{2} / T\right)$, where $\kappa=\epsilon_{3}^{(2)} / \epsilon_{5}$ and $\epsilon_{5} \neq 0$. Note that in even-numbered spans, the value of $\kappa$ is used for further stabilization of transmission and switching.

Transmission stabilization and switching are guided by stability analysis of the steady states of Eqs. (4) and (5). We therefore turn to describe the results of this analysis, starting with the LV model (4). We consider the equilibrium amplitude values $a=1$ and $b=\eta$, for which the linear gain coefficients are $g_{1}^{(1)}=4 \epsilon_{3}^{(1)}(1 / 3+2 \eta / T)$ and $g_{2}^{(1)}=4 \epsilon_{3}^{(1)}\left(\eta^{2} / 3+2 / T\right)$. We first note that $(1, \eta)$ is asymptotically stable, if $\eta>9 / T^{2}$, and is unstable otherwise. That is, $(1, \eta)$ undergoes a bifurcation at $\eta_{b i f}=9 / T^{2}$. The off-on and on-off switching are based on this bifurcation, and are realized dynamically by appropriate changes in linear amplifier gain. To explain this, we denote by $\eta_{t h}$ the value of the decision level, distinguishing between 
on and off transmission states. The off-on switching is achieved by a fast increase in $\eta$ from $\eta_{i}<\eta_{b i f}$ to $\eta_{f}>\eta_{b i f}$, such that the steady state $(1, \eta)$ turns from unstable to asymptotically stable. Consequently, before switching, $\eta_{1}$ and $\eta_{2}$ tend to $\eta_{s 1}>\eta_{t h}$ and $\eta_{s 2}<\eta_{t h}$, while after switching, $\eta_{1}$ and $\eta_{2}$ tend to 1 and $\eta>\eta_{t h}$. Thus, transmission of sequence 2 is turned on in this case. On-off switching is realized in a similar manner by a fast decrease in $\eta$ from $\eta_{i}>\eta_{b i f}$ to $\eta_{f}<\eta_{b i f}$. In this case $\eta_{1}$ and $\eta_{2}$ tend to 1 and $\eta>\eta_{t h}$ before the switching, and to $\eta_{s 1}>\eta_{t h}$ and $\eta_{s 2}<\eta_{t h}$ after switching. As a result, transmission of sequence 2 is turned off by the change in $\eta$.

Our coupled-NLS simulations show that stable ultra-long-distance transmission requires $T$ values larger than 15 . Indeed, for smaller $T$ values, high-order effects that are neglected by Eqs. (4) and (5), such as intrasequence interaction and radiation emission, lead to pulse pattern degradation and to breakdown of the LV model description at large distances. To enable comparison with results of Ref. [19] we choose $T=20$, but emphasize that similar results are obtained for other $T$ values satisfying $T>15$. For $T=20$, bifurcation occurs at $\eta_{b i f}=0.0225$. In transmission stabilization and off-on switching we use $\eta=1>\eta_{b i f}$, a choice corresponding to typical amplitude setups in many soliton-based transmission systems [2, 16]. In on-off switching, we use $\eta=0.02<\eta_{b i f}$. Note that the small $\eta$ value here is dictated by the small value of $\eta_{b i f}$.

Let us describe in some detail stability and bifurcation analysis for the equilibrium states of Eq. (41), for parameter values $a=1, b=\eta$, and $T>15$, which are used in both transmission stabilization and switching [46]. For these parameter values, the system (44) can have up to five steady states, located at $(1, \eta),(0,0),\left(A_{\eta}, 0\right),\left(0, B_{\eta}\right)$, and $\left(C_{\eta}, D_{\eta}\right)$, where $A_{\eta}=(1+6 \eta / T)^{1 / 2}, B_{\eta}=\left(\eta^{2}+6 / T\right)^{1 / 2}$,

$$
C_{\eta}=\left[\frac{-q(\eta)}{2}+\left(\frac{q^{2}(\eta)}{4}+\frac{p^{3}(\eta)}{27}\right)^{\frac{1}{2}}\right]^{\frac{1}{3}}+\left[\frac{-q(\eta)}{2}-\left(\frac{q^{2}(\eta)}{4}+\frac{p^{3}(\eta)}{27}\right)^{\frac{1}{2}}\right]^{\frac{1}{3}}-\frac{1}{3}
$$

$D_{\eta}=\eta+T\left(1-C_{\eta}^{2}\right) / 6, p(\eta)=-12 \eta / T-4 / 3$, and $q(\eta)=-16 / 27-8 \eta / T+216 / T^{3}$. Note that the first four equilibrium states exist for any $\eta>0$ and $T>0$. For $T>15$, the equilibrium state $\left(C_{\eta}, D_{\eta}\right)$ exists provided that $h_{1}(\eta)>0$, where $h_{1}(\eta)=(1+6 \eta / T)^{1 / 2}-C_{\eta}$. As mentioned earlier, the state $(1, \eta)$ is asymptotically stable if $\eta>9 / T^{2}$ and is unstable otherwise. In contrast, the state $(0,0)$ is unstable for any $\eta>0$ and $T>0$. The state $\left(A_{\eta}, 0\right)$ is asymptotically stable if $f_{1}(\eta)=(\eta T)^{3} / 36+\eta T^{2} / 3-6<0$ and is unstable otherwise, while $\left(0, B_{\eta}\right)$ is asymptotically stable for $f_{2}(\eta)=T^{3} / 36+\eta T^{2} / 3-6<0$ and is unstable otherwise. 
Finally, the steady state $\left(C_{\eta}, D_{\eta}\right)$ is asymptotically stable if $h_{2}(\eta)>0$ and $h_{3}(\eta)<0$, where $h_{2}(\eta)=\left(1 / 3+2 \eta / T-2 D_{\eta} / T-C_{\eta}^{2}\right)\left(\eta^{2} / 3+2 / T-2 C_{\eta} / T-D_{\eta}^{2}\right)-4 C_{\eta} D_{\eta} / T^{2}$ and $h_{3}(\eta)=\left(1+\eta^{2}\right) / 3+2(1+\eta) / T-2\left(C_{\eta}+D_{\eta}\right) / T-\left(C_{\eta}^{2}+D_{\eta}^{2}\right)$.

We now describe the phase portraits of Eq. (44), for the parameter values used in our coupled-NLS simulations. For the set $a=1, b=\eta=1$, and $T=20$, used in transmission stabilization and off-on switching, Eq. (44) has four steady states at $(1,1),(0,0),(\sqrt{1.3}, 0)$, and $(0, \sqrt{1.3})$, of which only $(1,1)$ is stable. In fact, as seen in the phase portrait of Eq. (4) in Fig. 1 (a), the steady state $(1,1)$ is globally asymptotically stable, i.e., the soliton amplitudes $\eta_{1}$ and $\eta_{2}$ both tend to 1 for any nonzero input amplitudes $\eta_{1}(0)$ and $\eta_{2}(0)$. The global stability of the steady state $(1,1)$ is crucial to the robustness of pulse control in hybrid waveguide setups, since it allows for transmission stabilization and off-on switching even for input amplitude values that are significantly smaller or larger than 1 . Furthermore, it can be used in broadband "transmission recovery", i.e., in the stable enhancement of pulse energies for multiple pulse sequences that experienced severe energy decay.

For the set $a=1, b=\eta=0.02$, and $T=20$, used in on-off switching, Eq. (4) has five steady states at $(1, \eta),(0,0),\left(0, B_{\eta}\right),\left(A_{\eta}, 0\right)$, and $\left(C_{\eta}, D_{\eta}\right)$, where $A_{\eta}=1.0030, B_{\eta}=0.5481$, $C_{\eta}=0.99925$, and $D_{\eta}=0.02502$. The first three states are unstable, while $\left(A_{\eta}, 0\right)$, and $\left(C_{\eta}, D_{\eta}\right)$ are asymptotically stable, as is also seen in the phase portrait of Eq. (4) in Fig. 1 (b). The asymptotic stability of $\left(A_{\eta}, 0\right)$ and $\left(C_{\eta}, D_{\eta}\right)$ along with their proximity to $(1,0)$ enable the switching off of transmission of sequence 2 for a wide range of input amplitude values.

Note that the instability of the steady state $(0,0)$ of Eq. (44), which is related to the presence of linear gain in the waveguide, is a major drawback of a uniform waveguide setup with linear gain and cubic loss. Indeed, the presence of linear gain leads to enhancement of small-amplitude waves that, coupled with modulational instability, can cause severe pulsepattern degradation. In the hybrid waveguide setup considered in the current paper, this instability is overcome by employing a GL gain-loss profile in even-numbered spans. We therefore turn to describe the results of stability analysis for the corresponding LV model (5). We choose $\eta=1$, and require $g_{j}^{(2)}<0$ for $j=1$, 2, i.e., the solitons propagate in the presence of net linear loss. Due to the linear loss, the steady state at $(0,0)$ is asymptotically stable, and as a result, energies of small-amplitude waves decay to zero, and pulse-pattern corruption is suppressed. In transmission stabilization and off-on switching stabilization, 
(a)

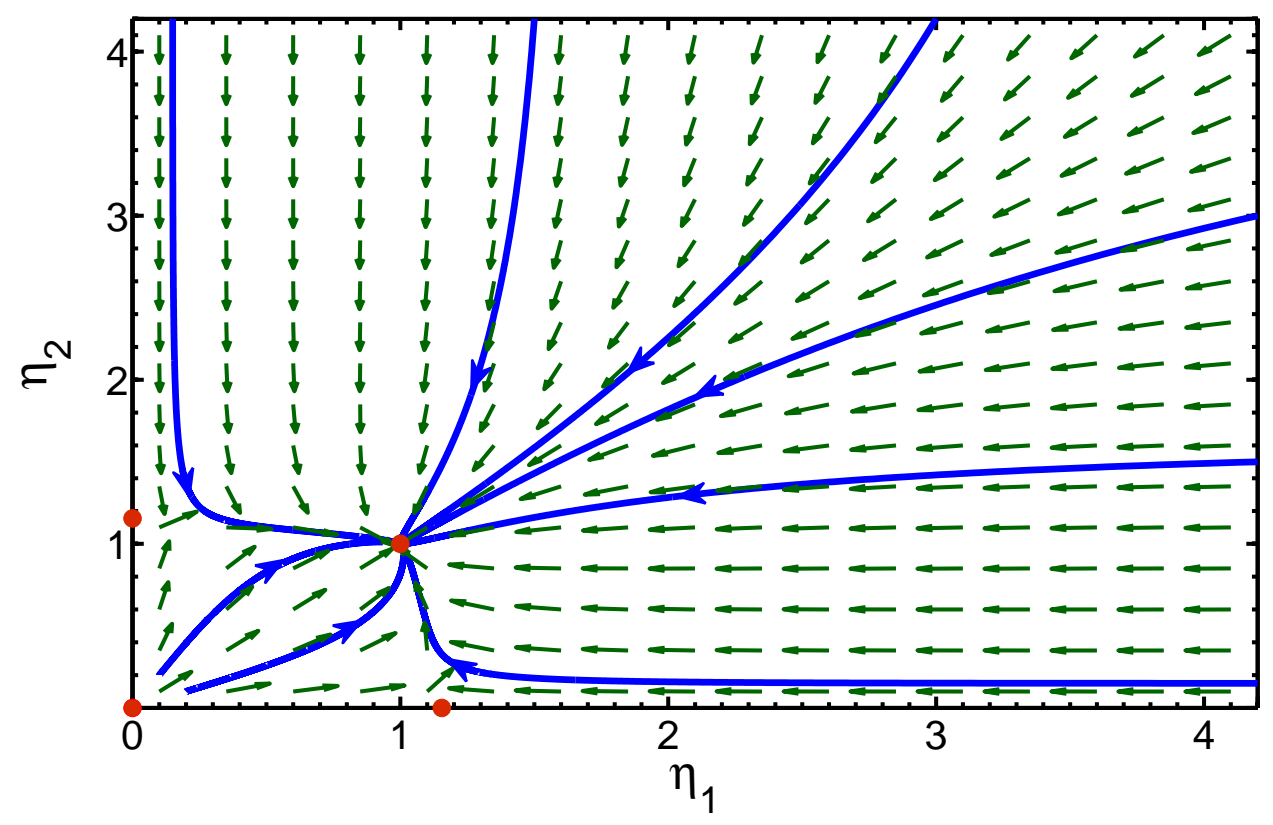

(b)

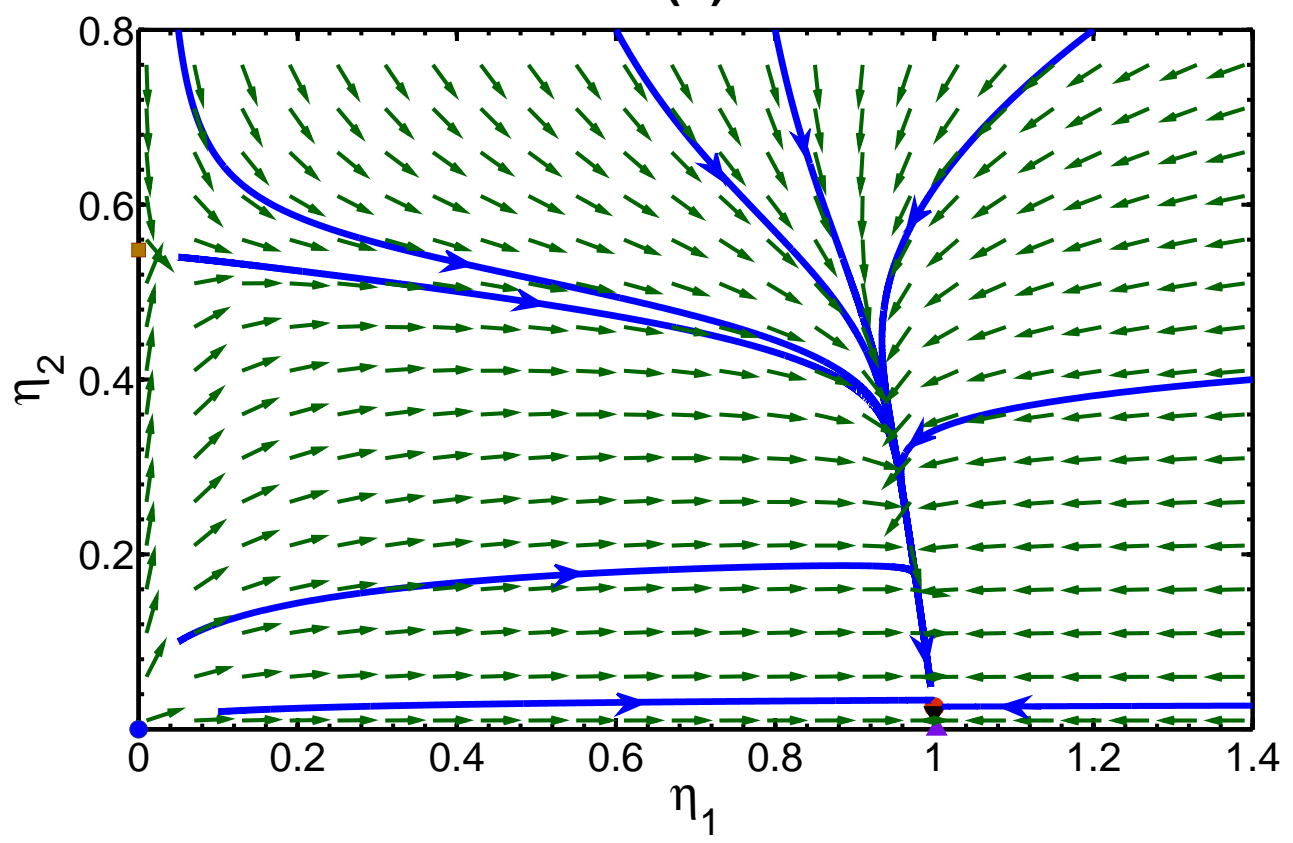

FIG. 1: (Color online) Phase portraits for the LV model (44) with parameter values $a=1, b=\eta=1$, $T=20$ in (a), and $a=1, b=\eta=0.02$, and $T=20$ in (b). The blue curves are numerically calculated trajectories. The four red circles in (a) correspond to the four equilibrium states. The red circle, black down triangle, magenta up triangle, blue circle, and orange square in (b) represent the equilibrium states at $\left(C_{\eta}, D_{\eta}\right),(1, \eta),\left(A_{\eta}, 0\right),(0,0)$, and $\left(0, B_{\eta}\right)$, respectively, where $A_{\eta}=1.0030$, $B_{\eta}=0.5481, C_{\eta}=0.99925$, and $D_{\eta}=0.02502$. 
we require that $(1,1)$ is an asymptotically stable steady state of Eq. (15). This requirement along with $g_{j}^{(2)}<0$ for $j=1,2$ yield the following condition [18]:

$$
(4 T+90) /(5 T+30)<\kappa<(8 T-15) /(5 T-15) \quad \text { for } \quad T \geq 60 / 17 .
$$

The values $\kappa=1.6$ and $T=20$ are used in coupled-NLS simulations of transmission stabilization, while $\kappa=1.65$ and $T=20$ are chosen in simulations of off-on switching stabilization. In stabilization of on-off switching we choose $T$ and $\kappa$ values satisfying

$$
\kappa>(8 T-15) /(5 T-15) \text { for } T \geq 60 / 17
$$

such that $(1,1)$ is unstable and another steady state at $\left(\eta_{s 1}, 0\right)$ is asymptotically stable. In this manner, the switching off of soliton sequence 2 is stabilized in even-numbered spans. In coupled-NLS simulations for on-off switching, $\kappa=2$ and $T=20$ are used and $\eta_{s 1}=$ 1.38255. We emphasize, however, that similar results are obtained for other values of $T$ and $\kappa$ satisfying $T>15$ and inequalities (6) or (7).

\section{NUMERICAL SIMULATIONS WITH THE HYBRID COUPLED-NLS MODEL}

The LV models (4) and (5) are based on several simplifying assumptions, whose validity might break down at intermediate-to-large propagation distances. In particular, the LV models neglect intrasequence interaction, radiation emission effects, and temporal inhomogeneities. These effects can lead to instabilities and pulse-pattern corruption, and also to the

breakdown of the LV description [17, 18]. In contrast, the coupled-NLS model (1) provides the full description of the propagation, which includes all these effects. Thus, in order to check whether long-distance transmission and robust broadband switching can be realized, it is important to carry out numerical simulations with the full coupled-NLS model.

The coupled-NLS system (1) is numerically solved using the split-step method with periodic boundary conditions [1]. The use of periodic boundary conditions means that the simulations describe propagation in a closed waveguide loop. The initial condition consists of two periodic sequences of $2 J+1$ overlapping solitons with amplitudes $\eta_{j}(0)$ and zero phase:

$$
\psi_{j}(t, 0)=\sum_{k=-J}^{J} \frac{\eta_{j}(0) \exp \left[i \beta_{j}(t-k T)\right]}{\cosh \left[\eta_{j}(0)(t-k T)\right]}
$$


where $j=1,2$, and $\beta_{1}=0, \beta_{2}=40, T=20$ and $J=2$ are used.

We first describe the results of numerical simulations for transmission stabilization. In this case we choose $a=1$ and $b=\eta=1$, so that the desired steady state of soliton amplitudes is $(1,1)$. We use two waveguide spans $[0,150)$ and $[150,2000]$ with gain-loss profiles consisting of linear gain and cubic loss in the first span, and of linear loss, cubic gain, and quintic loss in the second span. The cubic loss coefficient in the first span is $\epsilon_{3}^{(1)}=0.015$. The quintic loss coefficient in the second span is $\epsilon_{5}=0.05$, and the ratio between cubic gain and quintic loss is $\kappa=\epsilon_{3}^{(2)} / \epsilon_{5}=1.6$. The z-dependence of $\eta_{j}$ obtained by numerical simulations with Eq. (1) for input amplitudes $\eta_{1}(0)=1.2$ and $\eta_{2}(0)=0.7$ is shown in Fig. 2. Also shown is the prediction of the LV models (4) and (5). The agreement between the coupled-NLS simulations and the prediction of the LV models is excellent, and both amplitudes tend to 1 despite of the fact that the input amplitude values are not close to 1. Furthermore, as can be seen from the inset, the shape of the soliton sequences is retained during the propagation. Similar results are obtain for other choices of input amplitude values. We emphasize that the distances over which stable propagation is observed are larger by factors of 11 and 2 compared with the distances for the uniform waveguide setups considered in Refs. [17] and [19]. Additionally, the range of input amplitude values for which stable propagation is observed is significantly larger for hybrid waveguides compared with uniform ones. We therefore conclude that transmission stabilization is significantly enhanced by employing the hybrid waveguides described in the current paper.

We now turn to describe numerical simulations for transmission switching. The off-on and on-off transmission of sequence 2 is dynamically realized in odd-numbered spans by abrupt changes in the value of $\eta$ at distances $z_{s(m+1)}$ satisfying $z_{2 m}<z_{s(m+1)}<z_{2 m+1}$. These changes correspond to changes in the linear gain coefficients $g_{1}^{(1)}=4 \epsilon_{3}^{(1)}(1 / 3+2 \eta / T)$ and $g_{2}^{(1)}=4 \epsilon_{3}^{(1)}\left(\eta^{2} / 3+2 / T\right)$. In off-on switching, $\eta=0.02$ for $z_{2 m} \leq z<z_{s(m+1)}$ and $\eta=1$ for $z_{s(m+1)} \leq z<z_{2 m+1}$, so that the steady state $(1, \eta)$ becomes asymptotically stable. In on-off switching, the same $\eta$ values are used in reverse order and $(1, \eta)$ becomes unstable. After switching, transmission is stabilized in even-numbered spans by a proper choice of $\kappa$. In off-on switching stabilization, $\kappa=1.65$ is used, so that $(1,1)$ is asymptotically stable. In on-off switching stabilization, $\kappa=2$ is used, so that $(1,1)$ is unstable and $(1.38255,0)$ is asymptotically stable.

The following two setups of consecutive transmission switching are simulated: (A) off- 


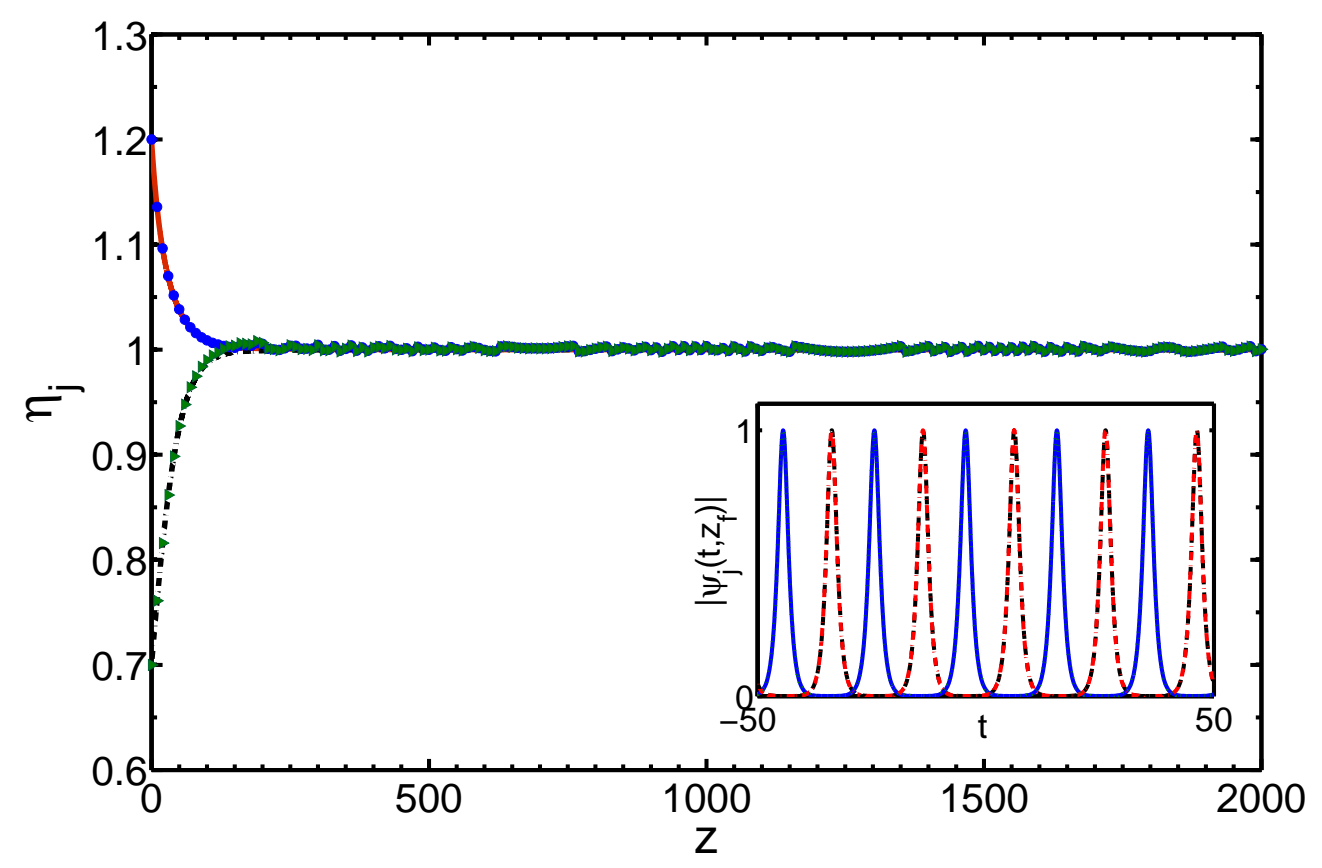

FIG. 2: (Color online) The z-dependence of soliton amplitudes $\eta_{j}$ for transmission stabilization with input amplitude values $\eta_{1}(0)=1.2$ and $\eta_{2}(0)=0.7$. The blue circles and green triangles represent $\eta_{1}(z)$ and $\eta_{2}(z)$ as obtained by numerical solution of Eq. (1), while the solid red and dashed-dotted black curves correspond to $\eta_{1}(z)$ and $\eta_{2}(z)$ values as obtained by the LV models (44) and (5). The inset shows the final pulse patterns. The dashed black and solid blue lines in the inset correspond to $\left|\psi_{1}\left(t, z_{f}\right)\right|$ and $\left|\psi_{2}\left(t, z_{f}\right)\right|$ obtained by numerical simulations with Eq. (11), while the dashed-dotted red and dotted green curves represent $\left|\psi_{1}\left(t, z_{f}\right)\right|$ and $\left|\psi_{2}\left(t, z_{f}\right)\right|$ obtained by summation over fundamental NLS solitons with unit amplitudes, frequencies $\beta_{1}=0$ and $\beta_{2}=40$, and positions $y_{j k}\left(z_{f}\right)$ for $j=1,2$ and $-2 \leq k \leq 2$, which were measured from the simulations.

on-off-on-off-on-off-on, (B) off-on-off-on-off-on-off. We emphasize that similar results are obtained with other transmission switching scenarios. The physical parameter values in setup A are $T=20, \epsilon_{3}^{(1)}=0.03, \epsilon_{5}=0.08$, and $\kappa_{(m+1)}=1.65$ for $0 \leq m \leq 3$. The waveguide spans are determined by $z_{2 m}=600 m$ for $0 \leq m \leq 4$ and $z_{2 m+1}=140+600 m$ for $0 \leq m \leq 3$. That is, the spans are $[0,140),[140,600), \ldots,[1800,1940)$, and $[1940,2400]$. The switching distances are $z_{s(m+1)}=100+600 m$ for $0 \leq m \leq 3$. The values of the physical parameters in setup B are the same as in setup A up to $z_{6}=1800$. At this distance, on-off switching is applied, i.e., $z_{s 4}=1800$. In addition, $z_{7}=1940, z_{8}=3000$, and $\kappa=2$ for $z_{7}<z \leq z_{8}$.

The results of numerical simulations with the coupled-NLS model (1) for setups A and 
$\mathrm{B}$ and input soliton amplitudes $\eta_{1}(0)=1.1$ and $\eta_{2}(0)=0.85$ are shown in Fig. 3 (a) and (b), respectively. A comparison with the predictions of the LV models (44) and (5) is also presented. The agreement between the coupled-NLS simulations and the predictions of the LV models is excellent for both switching scenarios. Furthermore, as shown in the inset of Fig. 3 (b), the shape of the solitons is preserved throughout the propagation and no growth of small amplitude waves (radiative instability) is observed. The propagation distances over which stable transmission switching is observed are larger by a factor of 3 compared with the distances reported in Ref. [19], even though in the current paper, seven and eight consecutive switching events are demonstrated compared with only one switching event in Ref. [19]. Moreover, off-on transmission switching is observed over a large range of amplitude values including $\eta_{2}$ values smaller than 0.35 . Consequently, the value of the decision level $\eta_{t h}$ for distinguishing between on and off states can be set as low as $\eta_{t h}=0.35$ compared with $\eta_{t h}=0.65$ for the uniform waveguides considered in Ref. [19]. Based on these observations we conclude that robustness of transmission switching is drastically increased in hybrid waveguide systems with a clever choice of the physical parameters. The increased robustness is a result of the global asymptotic stability of the steady state $(1,1)$ for the LV model (4), which is used to bring amplitude values close to their desired steady state values, and the local asymptotic stability of $(1,1)$ for the LV model (5), which is employed to stabilize the transmission against growth of small-amplitude waves.

\section{CONCLUSIONS}

In summary, we developed a method for transmission stabilization and switching for colliding sequences of optical solitons in broadband waveguide systems with nonlinear loss or gain. The method is based on employing hybrid waveguides, consisting of odd-numbered spans with linear gain and cubic loss, and even-numbered spans with a GL gain-loss profile, where the switching is dynamically realized by fast changes in linear amplifier gain.

We showed that dynamics of soliton amplitudes can be described by a hybrid LV model. Stability and bifurcation analysis of the steady states of the LV model was used to guide the choice of physical parameters values, which leads to a drastic enhancement in transmission stability and switching robustness. More specifically, the global asymptotic stability of the steady state $(1,1)$ of the LV model in odd-numbered spans was used to bring ampli- 
(a)

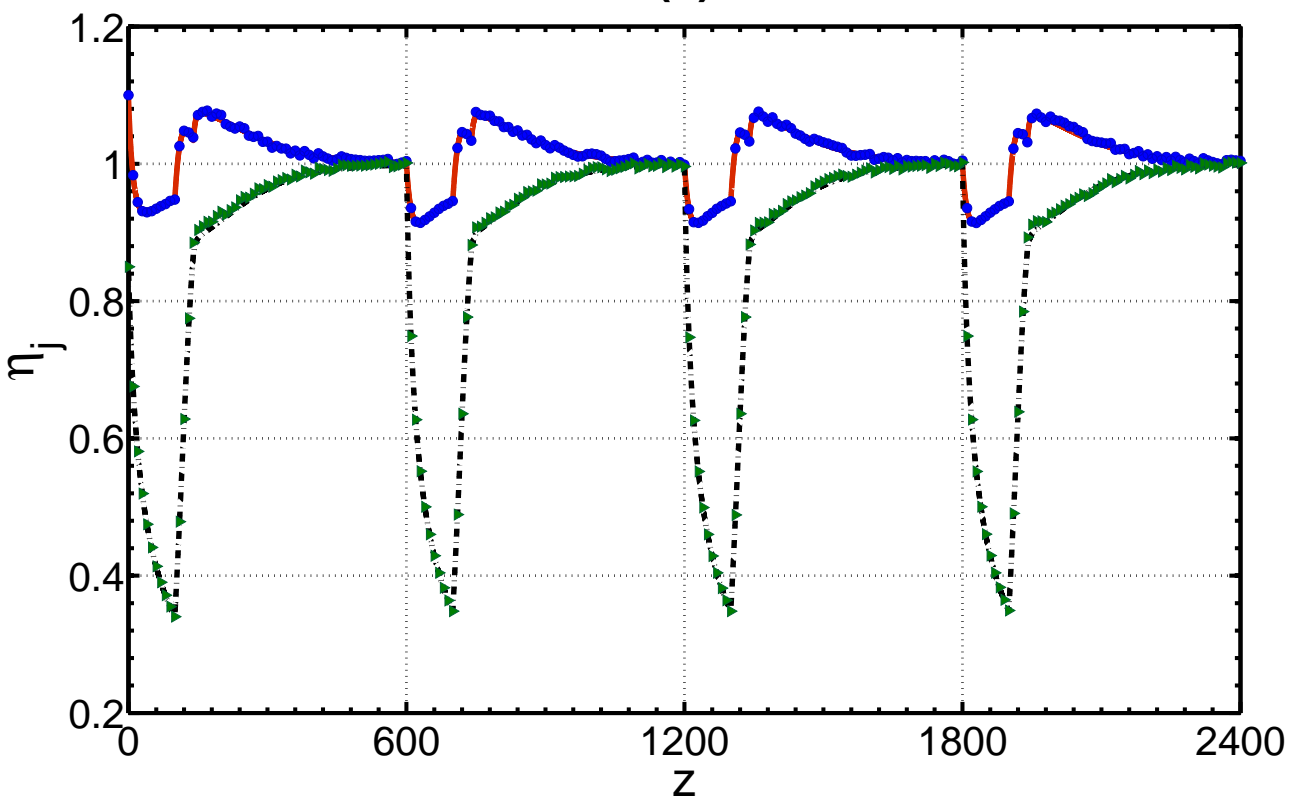

(b)

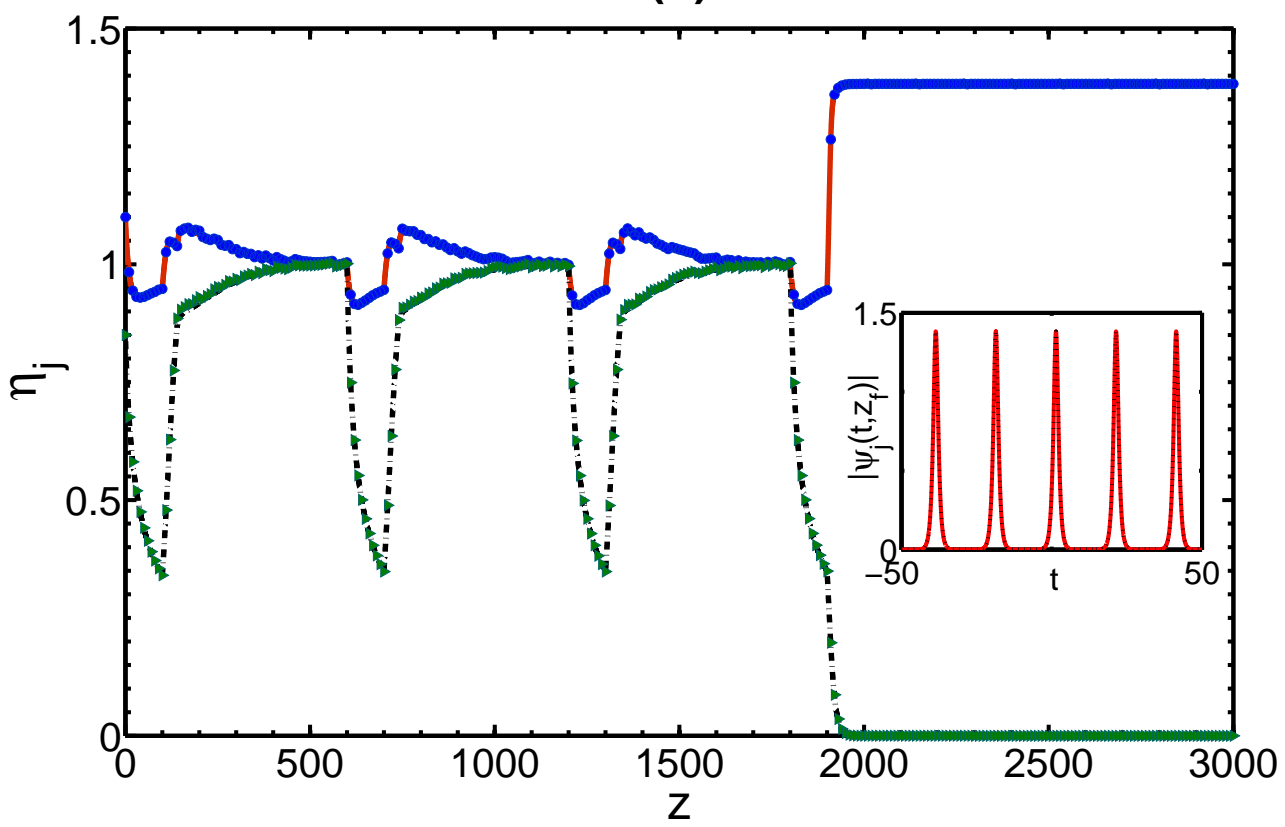

FIG. 3: (Color online) The z-dependence of soliton amplitudes $\eta_{j}$ in multiple transmission switching setups A (a) and B (b). The blue circles and green triangles represent $\eta_{1}(z)$ and $\eta_{2}(z)$ as obtained by numerically solving Eq. (1), while the solid red and dashed-dotted black curves correspond to $\eta_{1}(z)$ and $\eta_{2}(z)$ predicted by the LV models (4) and (5). The inset shows the final pulse pattern of pulse sequence $1\left|\psi_{1}\left(t, z_{f}\right)\right|$ in setup B, as obtained by numerical solution of Eq. (11) (solid red curve) and by summation over fundamental NLS solitons (dotted black curve) with amplitude $\eta_{1}=1.38255$, frequency $\beta_{1}=0$, and positions $y_{1 k}\left(z_{f}\right)$ for $-2 \leq k \leq 2$, which were measured from the simulations. 
tude values close to their desired steady state values, while the local asymptotic stability of the LV model in even-numbered spans was employed to stabilize the transmission against higher-order instability due to growth of small-amplitude waves. Numerical simulations with the coupled-NLS equations confirmed the predictions of the hybrid LV model. In particular, the simulations showed complete suppression of radiative instability due to growth of small amplitude waves, which enabled stable propagation over distances larger by an order of magnitude compared with the results reported in Ref. [17] for transmission in uniform waveguides with linear gain and cubic loss. Moreover, multiple on-off and off-on dynamic switching events, which are realized by fast changes in linear amplifier gain, were demonstrated over a wide range of soliton amplitudes, including amplitude values smaller than 0.35. As a result, the value of the decision level for distinguishing between on and off transmission states can be set as low as $\eta_{t h}=0.35$, compared with $\eta_{t h}=0.65$ for the single static switching event that was demonstrated in Ref. [19] in uniform waveguides with a GL gain-loss profile. Note that the increased flexibility in off-on switching in hybrid waveguides can be used for transmission recovery, i.e., for the stable amplification of soliton sequences, which experienced significant energy decay, to a desired steady state energy value. Based on these results, we conclude that the hybrid waveguide setups studied in the current paper lead to significant enhancement of transmission stability and switching robustness compared with the uniform nonlinear waveguides considered earlier.

Finally, it is worth making some remarks about potential applications of hybrid waveguides with different crosstalk mechanisms than the ones considered in the current paper. Of particular interest are waveguide setups, where the main crosstalk mechanism in oddnumbered and even-numbered spans are due to delayed Raman response and a GL gain-loss profile, respectively. One can envision employing these hybrid waveguides for enhancement of supercontinuum generation. Indeed, the interplay between Raman-induced energy exchange in soliton collisions and the Raman self-frequency shift is known to play a key role in widening the bandwidth of the radiation [47-51]. However, the process is somewhat limited due to the fact that energy is always transferred from high-frequency components to lowfrequency ones [1]. This limitation can be overcome by employing waveguide spans with a GL gain-loss profile subsequent to spans with delayed Raman response. Indeed, the main effect of cubic gain on soliton collisions is an energy increase for both high- and low-frequency solitons. As a result, the energies of the high frequency components of the radiation will 
be replenished in even-numbered spans. This will in turn sustain the supercontinuum generation along longer propagation distances and might enable a wider radiation bandwidth compared with the one in uniform waveguides, where delayed Raman response is the main crosstalk-inducing mechanism.

\section{Acknowledgments}

Q.M. Nguyen and T.P. Tran are supported by the Vietnam National Foundation for Science and Technology Development (NAFOSTED) under grant number 101.02-2012.10.

[1] G.P. Agrawal, Nonlinear Fiber Optics (Academic, San Diego, CA, 2001).

[2] L.F. Mollenauer and J.P. Gordon Solitons in Optical Fibers: Fundamentals and Applications (Academic, San Diego, CA, 2006).

[3] A.H. Gnauck, R.W. Tkach, A.R. Chraplyvy, and T. Li, J. Lightwave Technol. 26, 1032 (2008).

[4] R.-J. Essiambre, G. Kramer, P.J. Winzer, G.J. Foschini, and B. Goebel, J. Lightwave Technol. 28, $662(2010)$.

[5] R. Soref, IEEE J. Sel. Top. Quantum Electron. 12, 1678 (2006).

[6] Q. Lin, O.J. Painter, and G.P. Agrawal, Opt. Express 15, 16604 (2007).

[7] R. Dekker, N. Usechak, M. Först, and A. Driessen, J. Phys. D 40, R249 (2007).

[8] M.A. Foster, A.C. Turner, M. Lipson, and A.L. Gaeta, Opt. Express 16, 1300 (2008).

[9] J. Chow, G. Town, B. Eggleton, M. Ibsen, K. Sugden, and I. Bennion, IEEE Photon. Technol. Lett. 8, 60 (1996).

[10] H. Shi, J. Finlay, G.A. Alphonse, J.C. Connolly, and P.J. Delfyett, IEEE Photon. Technol. Lett. 9, 1439 (1997).

[11] J. Sun, Y. Zhang, and X. Zhang, IEEE Photon. Technol. Lett. 14, 750 (2002).

[12] H. Zhang, D.Y. Tang, X. Wu, and L.M. Zhao, Opt. Express 17, 12692 (2009).

[13] S.H. Oh, J.-U. Shin, K.S. Kim, D.-H. Lee, S.-H. Park, H.-K. Sung, Y.-S. Baek, and K.-R. Oh, Opt. Express 17, 9401 (2009).

[14] X. Liu, D. Han, Z. Sun, C. Zeng, H. Lu, D. Mao, Y. Cui, and F. Wang, Sci. Rep. 3, 2718 (2013). 
[15] F. Forghieri, R.W. Tkach, and A.R. Chraplyvy, in Optical Fiber Telecommunications III, I.P. Kaminow and T.L. Koch, eds., pp. 196-264, (Academic, San Diego, CA, 1997).

[16] E. Iannone, F. Matera, A. Mecozzi, and M. Settembre, Nonlinear Optical Communication Networks (Wiley, New York, 1998).

[17] A. Peleg, Q.M. Nguyen, and Y. Chung, Phys. Rev. A 82, 053830 (2010).

[18] A. Peleg and Y. Chung, Phys. Rev. A 85, 063828 (2012).

[19] D. Chakraborty, A. Peleg, and J.-H. Jung, Phys. Rev. A 88, 023845 (2013).

[20] R.W. Boyd, Nonlinear Optics (Academic, San Diego, CA, 2008).

[21] G.S. He, L.-S. Tan, Q. Zheng, and P.N. Prasad, Chem. Rev. 108, 1245 (2008).

[22] Y.S. Kivshar and B.A. Malomed, Rev. Mod. Phys. 61, 763 (1989).

[23] V. Mizrahi, K.W. DeLong, G.I. Stegeman, M.A. Saifi, and M.J. Andrejco, Opt. Lett. 14, 1140 (1989).

[24] Y. Silberberg, Opt. Lett. 15, 1005 (1990).

[25] A.B. Aceves and J.V. Moloney, Opt. Lett. 17, 1488 (1992).

[26] G.S. He, J.D. Bhawalkar, C.F. Zhao, and P.N. Prasad, Appl. Phys. Lett. 67, 2433 (1995).

[27] V.V. Afanasjev, J.S. Aitchison, and Y.S. Kivshar, Opt. Commun. 116, 331 (1995).

[28] J.E. Ehrlich, X.L. Wu, I.-Y.S. Lee, Z.-Y. Hu, H. Röckel, S.R. Marder, and J.W. Perry, Opt. Lett. 22, 1843 (1997).

[29] J. Zhang, Q. Lin, G. Piredda, R.W. Boyd, G.P. Agrawal, and P.M. Fauchet, Opt. Express 15, $7682(2007)$.

[30] W. Ding, C. Benton, A.V. Gorbach, W.J. Wadsworth, J.C. Knight, D.V. Skryabin, M. Gnan, M. Sorrel, and R.M. De La Rue, Opt. Express 16, 3310 (2008).

[31] G.S. He, Q. Zheng, K.-T. Yong, F. Erogbogbo, M.T. Swihart, and P.N. Prasad, Nano Lett. 8, 2688 (2008).

[32] Y. Okawachi, O. Kuzucu, M.A. Foster, R. Salem, A.C. Turner-Foster, A. Biberman, N. Ophir, K. Bergman, M. Lipson, and A.L. Gaeta, IEEE Photon. Technol. Lett. 24, 185 (2012).

[33] J.D. Moores, Opt. Commun. 96, 65 (1993).

[34] F.I. Khatri, J.D. Moores, G. Lenz, and H.A. Haus, Opt. Commun. 114, 447 (1995).

[35] N.N. Akhmediev, V.V. Afanasjev, and J.M. Soto-Crespo, Phys. Rev. E 53, 1190 (1996).

[36] I.S. Aranson and L. Kramer, Rev. Mod. Phys. 74, 99 (2002).

[37] J.N. Kutz, SIAM Review 48, 629 (2006). 
[38] W.H. Renninger, A. Chong, and F.W. Wise, J. Opt. Soc. Am. B 27, 1978 (2010).

[39] A. Peleg, Q.M. Nguyen, and P. Glenn, Phys. Rev. E 89, 043201 (2014).

[40] J.S. Aitchison, A.M. Weiner, Y. Silberberg, M.K. Oliver, J.L. Jackel, D.E. Leaird, E.M. Vogel, and P.W.E. Smith, Opt. Lett. 15, 471 (1990).

[41] O. Katz, Y. Lahini, and Y. Silberberg, Opt. Lett. 33, 2830 (2008).

[42] H.H. Fan, Y.J. He, J.W. Dong, B.C. Chen, H.Z. Wang, Y.P. Tian, and M.F. Reid, Appl. Phys. Lett. 96, 021109 (2010).

[43] C. Husko, S. Combrié, P. Colman, J. Zheng, A. De Rossi and C.W. Wong, Sci. Rep. 3, 1100 (2013).

[44] S. Novikov, S.V. Manakov, L.P. Pitaevskii, and V.E. Zakharov, Theory of Solitons: The Inverse Scattering Method (Plenum, New York, 1984).

[45] Q.M. Nguyen and A. Peleg, Opt. Commun. 283, 3500 (2010).

[46] Note that, as described below, the results of our bifurcation analysis for the four equilibrium states $(1, \eta),(0,0),\left(A_{\eta}, 0\right)$, and $\left(0, B_{\eta}\right)$ are valid for any $\eta>0$ and $T>0$.

[47] M.H. Frosz, O. Bang, and A. Bjarklev, Opt. Express 14, 9391 (2006).

[48] F. Luan, D.V. Skryabin, A.V. Yulin, and J.C. Knight, Opt. Express 14, 9844 (2006).

[49] N. Korneev, E.A. Kuzin, B. Ibarra-Escamilla, M. Bello-Jiménez, and A. Flores-Rosas, Opt. Express 16, 2636 (2008).

[50] E.R. Andresen, J.M. Dudley, D. Oron, C. Finot, and H. Rigneault, J. Opt. Soc. Am. B 28, 1716 (2011).

[51] M. Andreana, A. Labruyère, A. Tonello, S. Wabnitz, P. Leproux, V. Couderc, C. Duterte, A. Cserteg, A. Bertrand, Y. Hernandez, D. Giannone, S. Hilaire, and G. Huss, Opt. Express 20, $10750(2012)$. 